\title{
2. Über die Struktur des endlichen, vollständig primären Ringes mit verschwindendem Radikalquadrat.
}

\author{
Von Kunihiko KODAIRA
}

(Eingegangen am 17. November, 1936.)

Es sei $\mathfrak{U}$ ein endlicher, vollständig primärer Ring, dessen Radikal $\Re$ der Bedingung:

$$
\Re^{2}=0
$$

genügt $\left({ }^{1}\right)$. In der vorliegenden Arbeit soll die Struktur solcher Ringe $\mathfrak{A}$ vollständig bestimmt werden.

1. Der Restklassenring

$$
K=\mathscr{U} / \Re
$$

ist ein endlicher Körper, also ein Galoisfeld $G F(q)$, wo $q$ eine Potenz einer Primzahl $p$ ist:

$$
q=p^{n} .
$$

Wegen $\Re^{2}=0$ folgt aus $a \equiv b(\bmod \Re)$, dass

$$
a r=b r, \quad r a=r b \quad \text { für jedes } r \text { aus } \Re
$$

ist. Das Produkt ar bzw. ra hängt also nur von der Restklasse $\alpha \in K$ von $\alpha \bmod \mathfrak{R}$ ab. Wir definieren das Produkt von $\alpha \in K$ und $r \in \mathfrak{R}$ durch die Festsetzung:

$$
\alpha r=a r, \quad r \alpha=r a .
$$

Dadurch wird $\Re$ ersichtlich ein $K$-K-Doppelmodul. Für ein Element $\pi$ aus dem Primkörper $P$ aus $K$ gilt dabei:

$$
\pi r=\underbrace{r+\cdots \cdots+r}_{\pi}=r \pi .
$$

$\Re$ bildet somit einen Darstellungsmodul von $K$ in sich, wobei $P$ als Grundkörper angenommen wird. $K$ ist nun ein separabler, galoisscher Erweiterungskörper von $P ; \Re$ ist also vollständig reduzibel und jeder irreduzible Bestandteil ist eingliedrig. Unter geeigneter Wahl der $K$-basis hat also $\mathfrak{R}$ die Form:

$$
\Re=r_{1} K+r_{2} K+\cdots \cdots+r_{s} K
$$

(1) Vgl. M. Deuring, Algebren (1935), S. 18. 
und es gilt dabei

$$
a r_{i}=r_{i} \alpha^{A i}, \quad \alpha \in K,
$$

wo $A_{i}$ einen Automorphismus von $K$ bedeutet.

Aus der Eindeutigkeit der direkten Summenzerlegung ergibt sich, dass die Anzahl der Basiselemente $r_{i}$, die den Automorphismus $A$ induzieren, von der speziellen Wahl der Basis nicht abhängt.

2. $\rho_{\ell}$ sei ein primitives Element von $K$ und $g_{1}$ ein zur Restklasse $\rho_{1}$ gehöriges Element aus $\mathfrak{A}$. Dann ist

$$
\rho_{1}^{q-1}=1,
$$

also

$$
g_{1}^{\alpha-1} \equiv e \quad(\bmod \Re)
$$

oder

$$
g_{1}^{\alpha-1}=e+r, \quad r \in \Re,
$$

wo $e$ das Einselement von $\mathfrak{A}$ ist. Somit ist

$$
\left(g_{1}^{p}\right)^{q-1}=\left(g_{1}^{q-1}\right)^{p}=(e+r)^{p}=e+p \cdot r=e .
$$

Nun gehört $g_{1}^{p}$ zur Restklasse $\rho=\rho_{1}^{p}$, und $\rho$ ist auch ein primitives Element von $K$. Setzen wir nun

so ist die Reihe

$$
g=g_{1}^{p}
$$

$$
0, e, g^{1}, g^{2}, \ldots \ldots g^{q-2}
$$

ein vollstandiges Restsystem mod. $\Re$ und zum System $0,1, \rho, \ldots \rho^{q-2}$ multiplikativ isomorph, weil $g^{q-1}=e$ ist.

Schreiben wir dann

so gilt

$$
g^{l}=g_{\alpha} \quad \text { für } \quad \alpha=\rho^{l},
$$

und zugleich

$$
g_{\alpha} g_{\beta}=g_{\alpha \beta}, \quad g_{1}=e,
$$

$$
g_{\alpha}+g_{\beta} \equiv g_{\alpha+\beta} \quad(\bmod \Re) \text {. }
$$

Das letztere lässt sich natürlich auch so schreiben

$$
g_{\alpha}+g_{\beta}=g_{\alpha+\beta}+r_{\alpha, \beta} \text { mit } r_{\alpha, \beta} \in \Re,
$$

wobei die $q^{2}$ Elemente $r_{\alpha, \beta}$ einigen Bedingungen genügen můssen :

Aus $g_{\alpha}+g_{\beta}=g_{\beta}+g_{\alpha}$ folgt nämlich

I. $r_{\alpha, \beta}=r_{\beta, \alpha}$; 
aus $g_{\mathrm{r}}\left(g_{\alpha}+g_{\beta}\right)=g_{\gamma} g_{\alpha}+g_{\gamma} g_{\beta}, \quad\left(g_{\alpha}+g_{\gamma}\right) g_{\gamma}=g_{\alpha} g_{\gamma}+g_{\beta} g_{r}$,

II. $\gamma \cdot r_{\alpha, \beta}=r_{\gamma \alpha, \gamma \beta}, \quad r_{\alpha, \beta} \circ \gamma=r_{\alpha \gamma, \beta \gamma}$,

und aus dem Assoziativgesetz $\left(g_{\alpha}+g_{\beta}\right)+g_{\uparrow}=g_{\alpha}+\left(g_{\beta}+g_{\gamma}\right)$,

III. $r_{\alpha+\beta, \gamma}+r_{\alpha, \beta}=r_{\alpha, \beta+\gamma}+r_{\beta, \gamma}$.

Jedes Element $a$ aus $\mathfrak{A}$ ist nun eindeutig als Summe der Gestalt

$$
a=g_{\alpha}+r, \quad r \in \Re,
$$

darstelltbar, und es gilt:

$$
\begin{aligned}
\left(g_{\alpha}+r\right)+\left(g_{\beta}+r^{\prime}\right) & =g_{\alpha+\beta}+\left(r_{\alpha, \beta}+r+r^{\prime}\right), \\
\left(g_{\alpha}+r\right)\left(g_{\beta}+r^{\prime}\right) & =g_{\alpha \beta}+\left(\alpha r^{\prime}+r \beta\right) .
\end{aligned}
$$

Also ist die Struktur von $\mathfrak{A}$ durch das System $r_{\alpha, \beta}$ vollständig bestimmt.

Umgekehrt sei $K$ ein Galoisfeld und $\Re$ ein $K$-K-Doppelmodul. Wenn man in $\Re$ den Bedingungen I, II, III, genügende Elemente $r_{\alpha, \beta}$ beliebig wählt, und Summen und Produkte zweier Elemente der Menge $\mathfrak{A}$ aller formalen Ausdrücke

$$
g_{\alpha}+r, \quad r \in \Re,
$$

durch die Formeln (1) und (2) definiert, so wird $\mathfrak{A}$ ein vollständig primärer Ring mit Radikal $\Re$, und es gilt dabei $K \cong \mathfrak{U} / \Re, \mathfrak{R}^{2}=0$.

3. Gemäss der in $\$ 1$ angegebenen Zerlegung von $\Re$ in direkte Summanden setzen wir nun

$$
r_{\alpha, \beta}=\sum_{i=1}^{s} r_{i} \phi_{i}(\alpha . \beta) \quad \text { mit } \phi_{i}(\alpha, \beta) \in K .
$$

Die Bedingungen I, II, III, im letzten Paragraphen sind dann mit folgenden Bedingungen für $\varphi_{i}$ äquivalent:

$$
\begin{aligned}
\mathrm{I}^{\prime} . & \phi_{i}(\alpha, \beta)=\phi_{i}(\beta, \alpha), \\
\mathrm{II}_{1}^{\prime} . & \gamma^{A_{i}} \phi_{i}(\alpha, \beta)=\phi_{i}(\gamma \alpha, \gamma \beta), \\
\mathrm{II}_{2 \cdot}^{\prime} & \phi_{i}(\alpha, \beta) \cdot \gamma=\phi_{i}(\alpha \gamma, \beta \gamma), \\
\mathrm{III}^{\prime} . & \phi_{i}(\alpha+\beta, \gamma)+\phi_{i}(\alpha, \beta)=\phi_{i}(\alpha, \beta+\gamma)+\phi_{i}(\beta, \gamma) .
\end{aligned}
$$

Aus $\mathrm{II}_{1}^{\prime}$ und $\mathrm{II}_{2}^{\prime}$ folgt nun

$$
\gamma^{A_{i}} \varphi_{i}(\alpha, \beta)=\varphi_{i}(\alpha, \beta) \gamma .
$$


Ist $A_{i}$ ein nicht-identischer Automorphismus; so ist

$$
\gamma^{A_{i}} \neq \gamma
$$

für geeignetes $\gamma$ aus $K$; also muss

$$
\varphi_{i}(\alpha, \beta)=0
$$

sein. Ist aber $A_{i}$ dex identische Automorphismus, so sind $\mathrm{II}_{1}^{\prime}$ und $\mathrm{II}_{2}^{p}$ äquivalent mit

II'.

$$
\gamma \varphi_{i}(\alpha, \beta)=\varphi_{i}(\gamma \alpha, \gamma \beta)
$$

4. Die Anzahl allex möglichen Funktionen von $n$ Variablen im Galoisfeld $G F(q)$ ist $q^{q^{n}}$. Die $q^{q^{n}}$ ganz-rationale Funktionen

$$
\sum_{0 \leq i_{y} \leq q-1} a_{i_{1} i_{2} \ldots i_{n}} x_{1}^{i_{1}} x_{2}^{i_{2}} \ldots x_{n}^{i_{n}}
$$

sind aber såmtlich verschieden. Also ist jede Funktion in $G F(q)$ ganzrational darstellbar.

Insbesondere lässt sich eine den Bedingungen I', II', III' genügende Funktion $\varphi(x, y)$ in der Form schreiben:

$$
\varphi(x, y)=\sum_{\substack{0 \leq i \leq q-1 \\ 0 \leq k \leq q-1}} \alpha_{i k} x^{i} y^{k},
$$

wobei den Koeffizienten $\alpha_{i k}$ gewisse Bedingungen auferlegt werden. Nach $I^{\prime}$ gìlt nämlich

und nach II'

$$
\alpha_{i k}=\alpha_{k i}
$$

$$
\sum \alpha_{i k} z x^{i} y^{k}=\sum \alpha_{i k} x^{i} y^{k} z^{i+k}
$$

oder, wegen $z^{q}=z$,

$$
\sum \alpha_{i k} z x^{i} y^{k}=\sum_{j=0}^{q-1} z^{j} \sum_{i+k=j} \alpha_{i k} x^{i} y^{k}+\sum_{j=1}^{q-1} z^{j} \sum_{i+k k_{m} j+(q-1)} \alpha_{i k} x^{i} y^{k}
$$

Durch Vergleich der Koeffizienten ergibt sich hieraus

$$
\alpha_{i k}=0, \quad \text { wenn } i+k \neq 1 \text { und } \neq q \text {. }
$$

$\varphi(x, y)$ hat also die Form

$$
\varphi(x, y)=\alpha_{0}(x+y)+\sum_{i=1}^{q-1} \alpha_{i} x^{i} y^{\alpha-i}
$$

wobei $\alpha_{0}=\alpha_{01}=\alpha_{10}, \quad \alpha_{i}=\alpha_{q-i}=\alpha_{i, q-i}=\alpha_{q-i, i}$ gesetzt ist. Wir können danach ausrechnen: 


$$
\begin{aligned}
\varphi(x+y, z)+\varphi(x, y)= & \alpha_{0}(2 x+2 y+z)+\sum_{i=1}^{q-1} \alpha_{i} \sum_{j=1}^{i-1}\left(\begin{array}{c}
i \\
j
\end{array}\right) x^{i-j} y^{j} z^{q-i} \\
& +\sum_{i=1}^{q-1} \alpha_{i}\left(x^{i} y^{q-i}+y^{i} z^{q-i}+z^{i} x^{q-i}\right)
\end{aligned}
$$

Aus Bedingung III' folgt nun, dass $\alpha_{0}=0$ und

$$
\left(\begin{array}{c}
q-\mu \\
\lambda
\end{array}\right) \alpha_{\mu}=\left(\begin{array}{c}
q-\lambda \\
\mu
\end{array}\right) \alpha_{\lambda} \quad \text { für } \lambda, \mu \geqq 1, \lambda+\mu \leqq q-1
$$

ist. Aus

$$
(x+1)^{p^{m}}=x^{p^{m}}+1 \quad(\bmod p)
$$

ergibt sich aber

$$
(x+1)^{p^{m-1}} \equiv x^{p^{m-1}}-x^{p^{m-2}}+\cdots \cdots \pm 1 \quad(\bmod p),
$$

also ist

$$
\left(\begin{array}{c}
p^{m}-1 \\
\nu
\end{array}\right) \equiv \pm 1 \quad(\bmod p)
$$

Aus

$$
(x+1)^{p^{m i}} \equiv\left(x^{p^{m}}+1\right)^{\lambda} \quad(\bmod p)
$$

erhält man ferner

$$
\begin{gathered}
\left(\begin{array}{c}
p^{m} \lambda \\
p^{m} \mu
\end{array}\right) \equiv\left(\begin{array}{l}
\lambda \\
\mu
\end{array}\right) \quad(\bmod p), \\
\left(\begin{array}{c}
p^{m} \lambda \\
\nu
\end{array}\right) \equiv 0 \quad(\bmod p), \quad \text { für } \nu \neq 0\left(\bmod p^{m}\right) .
\end{gathered}
$$

Nun setzen wir in (6) $\mu=p^{m}, m \leqq n-2$ und $\lambda=p^{n-1}$, so haben wir

$$
\left(\begin{array}{c}
p^{n}-p^{m} \\
p^{n-1}
\end{array}\right) \alpha_{p^{m}}=\left(\begin{array}{c}
p^{n}-p^{n-1} \\
p^{m}
\end{array}\right) \alpha_{p^{n-1}}
$$

Wegen $(7),(8),(9)$ muss also

$$
\alpha_{p^{m}}=0 \quad(m \leqq n-2)
$$

sein.

Man setze nun in (6) $\mu=\mu_{0} p^{m}, m \leqq n-2,\left(\mu_{0}, p\right)=1$, dann gilt

$$
\left(\begin{array}{c}
p^{n}-\mu_{0} p^{m} \\
p^{m}
\end{array}\right) \alpha_{u_{0} p^{m}}=\left(\begin{array}{c}
p^{n}-p^{m} \\
\mu_{0} p^{m}
\end{array}\right) \alpha_{p^{m}}
$$

also nach (10) 


$$
\left(\begin{array}{c}
p^{n-m}-\mu_{0} \\
1
\end{array}\right) \alpha_{\mu_{0} p^{m}}=0,
$$

d. h.

$$
\alpha_{\mu_{0} p^{m}}=0 \text {. }
$$

Für $\quad \mu=\mu_{0} p^{n-1}, \quad \lambda=\lambda_{0} p^{n-1}$ ist

oder

$$
\left(\begin{array}{c}
p^{n}-\mu_{0} p^{n-1} \\
\lambda_{0} p^{n-1}
\end{array}\right) \alpha_{\mu_{0} p^{n-1}}=\left(\begin{array}{c}
p^{n}-\lambda_{0} p^{n-1} \\
\mu_{\nu} p^{n-1}
\end{array}\right) \alpha_{\lambda_{\gamma_{0}} p^{n-1}},
$$

$$
\left(\begin{array}{c}
p-\mu_{0} \\
\lambda_{0}
\end{array}\right) \alpha_{\mu_{0} p^{n-1}}=\left(\begin{array}{c}
p-\lambda_{0} \\
\mu_{0}
\end{array}\right) \alpha_{\lambda_{0} p^{n-1}} .
$$

Danach erhält man

$$
\alpha_{u_{0} p^{n-1}}=\frac{(p-1) !}{\mu_{0} !\left(p-\mu_{0}\right) !} \alpha_{p^{n-1}} .
$$

Nach (11) und (12) muss die Funktion $\varphi(x, y)$ schliesslich die Form

$$
\phi(x, y)=\alpha \cdot \sum_{\mu=1}^{p-1} \frac{(p-1) !}{\mu !(p-\mu) !} x^{p n-1 \mu} y^{p n-1}(p-\mu)
$$

haben. Umgekehrt genügt diese Funktion offenbar den Bedingungen $I^{\prime}$, II', III'。

5. Setzen wir also

$$
\psi(x, y)=\sum_{\mu=1}^{p-1} \frac{(p-1) !}{\mu !(p-\mu) !} x^{p^{n-1} \mu} y^{p^{n-1}(p-\mu)},
$$

so können wir

und

$$
\varphi_{i}(\alpha, \beta)=\alpha_{i} \psi(\alpha, \beta)
$$

$$
r_{\alpha, \beta}=\sum_{i} r_{i} \alpha_{i} \cdot \psi(\alpha, \beta)
$$

schreiben, wobei $r_{i}$ die den identischen Automorphismus induzierenden Basisselemente durchläuft. Wenn $r^{*}=\sum_{i} r_{i} \alpha_{i} \neq 0$ ist, können wir $r^{*}$ als ein Basiselement $r_{1}$ von $\Re$ annehmen; dann wird

Andernfalls ist

$$
r_{\alpha_{0} \beta}=r_{1} \cdot \psi(\alpha, \beta) \text {. }
$$

$$
r_{\alpha, \beta}=0 \quad \text { für alle } \alpha, \beta \text {. }
$$

Damit ist folgender Satz bewiesen:

Satz 1. Jedes Element a aus $\mathfrak{A}$ ist eindeutig als die Summe: 


$$
a=g_{\alpha}+\sum_{i=1}^{s} r_{i} \alpha_{i}, \quad \alpha_{,} \alpha_{i} \in K,
$$

darstellbar. Dabei ist das Produkt bzw. die Summe zweier Elemente nach der Regel gebildet:

$$
\begin{gathered}
\left(g_{\alpha}+\sum_{i=1}^{s} r_{i} \alpha_{i}\right)\left(g_{\beta}+\sum_{i=1}^{s} r_{i} \beta_{i}\right)=g_{\alpha \beta}+\sum_{i=1}^{s} r_{i}\left(\alpha^{A} \beta_{i}+\beta \alpha_{i}\right) \\
\left(g_{\alpha}+\sum_{i=1}^{s} r_{i} \alpha_{i}\right)+\left(g_{\beta}+\sum_{i=1}^{s} r_{i} \beta_{i}\right)=g_{t+\beta}+r_{1}\left(\phi(\alpha, \beta)+\alpha_{1}+\beta_{1}\right)+\sum_{i=2}^{s} r_{i}\left(\alpha_{i}+\beta_{i}\right)
\end{gathered}
$$

wobei $A_{i}$ Automorphismus von $K$ bedeutet, und $A_{1}=1$. Die Funktion $\phi(\alpha, \beta)$ ist entweder identisch gleich $N u l l$, oder gleich der oben angegebenen Funktion $\psi(\alpha, \beta)$.

6. Ist $\varphi=0$, bildet die Gesamtheit aller $g_{\alpha}$ einen zum $K$ isomorphen Teilkörper von $\mathfrak{A}$. Im Fall $\varphi=\psi$ enthält $\mathfrak{U}$ dagegen keinen Teilkörper mit dem Einselement $e$; denn die additive Ordnung von $e$ ist dann $p^{2}$, also keine Primzahl ; in der Tat ist der Restklassenring des Ringes der ganz-rationalen Zahlen $\left(\bmod p^{2}\right)$ ein Ring mit $\varphi=\psi$, und die durch $\psi$ eindeutig bestimmte Summe $p \cdot e$ ist darin nicht gleich Null. Also sind der Ring mit $\phi=\psi$ und der Ring mit $\varphi=0$ sicher nicht zueinander isomorph. Es gilt also der

$\mathrm{S}_{\text {atz }}$ 2. Die Funktion $\varphi$ und die Anzahl $s_{A}$ der zu A gehörigen Basiselemente $r_{i}$ sind durch die Struktur von $\mathfrak{A}$ eindeutig bestimmt. Umgelehrt ist die Struktur von $\mathfrak{A}$ durch $\varphi$ und $s_{A}$ eindeutig bestimmt.

Ist $K$ insbesondere ein Primkörper, so gibt es keinen anderen Automorphismus als den identischen; also ist $\mathfrak{A}$ kommutativ. 